\title{
Unexpected diagnosis for a gastric polyp: Granular cell tumor: Case report and review of the literature
}

\author{
SORINA MARIA TABAN ${ }^{1}$, ROBERT ALEXANDRU BARNA ${ }^{2,3}$, ALIS LILIANA CARMEN DEMA ${ }^{1}$, \\ IULIA MARIA RATIU ${ }^{2}$, OANA POPA ${ }^{1,4}$ and ANDREI DOREL PLOPEANU ${ }^{3}$ \\ ${ }^{1}$ Department of Microscopic Morphology-Morphopathology; ${ }^{2}$ Department of Internal Medicine \\ II, Discipline of Gastroenterology and Hepatology, 'Victor Babeș' University of Medicine and \\ Pharmacy, Timisoara, 300041 Timisoara; ${ }^{3}$ Anatomic Pathology Service, ${ }^{4}$ Endocrinology Clinic, \\ 'Pius Brînzeu' County Emergency Clinical Hospital, 300723 Timisoara, Romania
}

Received January 4, 2021; Accepted February 3, 2021

DOI: $10.3892 / e t m .2021 .9968$

\begin{abstract}
A granular cell tumor (GCT) is a rare neoplasia that originates from Schwann cells. It usually appears in the skin or soft tissues, but it may occur anywhere in the body. The gastrointestinal tract is an unusual developmental site for a GCT, the esophagus being the most common site of origin for this tumor. The stomach is one of the most unique sites of origin for GCT, with less than 80 cases being mentioned in the literature. Histologically, GCTs consist of fusiform and polygonal cells, with granular cytoplasm, arranged in compact 'nests'. Immunohistochemically, these tumors show positivity for S100 protein, CD68, CD56 and, in a smaller percentage, they are positive for other antibodies, most notably inhibin alpha. We report the case of a 52-year-old woman with a solitary GCT that had developed in the gastric cardia, discovered on a routine gastroscopy and successfully treated by endoscopic submucosal dissection.
\end{abstract}

\section{Introduction}

Granular cell tumor (GCT) was initially discovered by Weber in 1854 (1), but more accurately reported by Abrikossoff in 1926 (2). GCT was initially called 'granular cell myoblastoma' due to the suspected muscle origin, but electronic microscopy and immunohistochemical (IHC) studies showed that GCTs are of Schwann cell origin (3-5).

The most frequently affected regions are the tongue, skin and subcutaneous tissues or breast (6). GCTs in the

Correspondence to: Dr Robert Alexandru Barna, Department of Internal Medicine II, Discipline of Gastroenterology and Hepatology, 'Victor Babeș' University of Medicine and Pharmacy, Timisoara, 2 Eftimie Murgu Square, 300041 Timisoara, Romania E-mail: barna.robert27@gmail.com

Key words: granular cell tumor, gastric cardia, S100, inhibin alpha, endoscopic submucosal dissection gastrointestinal (GI) tract are uncommon (approximately 8\% of all GCTs), mostly affecting the esophagus or colon and less frequently the stomach $(7,8)$. Most GCTs are benign, with fewer than $2 \%$ of cases being malignant (9).

Histologically, GCTs are made up of large polygonal and fusiform cells, consisting of an expansive and granular cytoplasm, arranged in nests. GCTs are almost always positive for S100 protein, CD68 and CD56 antibodies. Endoscopic examination of the lesion reveals more commonly a yellowish-white sessile mass, covered by normal appearing mucosa (3).

Herein, we report an incidental GCT that had developed in the gastric cardia and successfully removed by endoscopic submucosal dissection (ESD). A review of the literature concerning gastric GCTs is also presented.

\section{Case report}

A 52-year-old woman presented to a gastroenterologist for routine colonoscopy on March, 2020. The patient complained over the past few months of upper abdominal discomfort after eating, without dysphagia or other distinct symptoms and was unable to mention when the symptoms commenced. Personal medical history was unremarkable and the family medical history included a distant relative with colorectal adenocarcinoma.

Upon examination, the colonoscopy showed no abnormalities, but upper GI endoscopy revealed a polypoid lesion of the gastric cardia. ESD of the lesion was performed and the specimen was submitted for histopathological evaluation.

Gross examination of the resected specimen revealed an elastic, tan, polypoid fragment of $12 \times 10 \times 8 \mathrm{~mm}$, with a stalk of 9x8 mm. The cut surface showed a solid, yellowish nodular tumor of $6 \times 5 \mathrm{~mm}$. Relevant sections including tumor with the stalk were sampled for histological examination.

Microscopic examination using hematoxylin and eosin (H\&E) staining showed a well-defined, nodular tumor, which developed into the submucosal layer and was covered by normal gastric mucosa. It was composed of spindle and polygonal cells, arranged in strands, with abundant eosinophilic 
granular cytoplasm. Nuclei were slightly pleomorphic and vesicular, without mitotic activity (Fig. 1A and B).

Tumor cells were positive for S-100 protein (Fig. 1C and D), inhibin alpha (Fig. 1E), CD56 (Fig. 1F) and CD68. CD34 expression was noted within the tumor, more prominent towards tumor borders, while the Ki-67 index was lower than $1 \%$. The entire panel of markers used for IHC analysis of this case is presented in Table I.

Surgical margins were tumor-free, with neoplastic cells located at $0.4 \mathrm{~mm}$ of the closest limit.

Based on the H\&E examination and IHC profile, the tumor was diagnosed as a benign GCT. The ESD was uneventful and the patient was discharged on the same day. At 5 months of follow-up, there were no signs of recurrence or other related events.

\section{Discussion}

GCT was initially reported by Weber in 1854 (1), but only 70 years later, in 1926, was this type of tumor properly described by Abrikosoff (2). Abrikossoff initially described GCT as a 'granular cell myoblastoma' because of suspected muscle origin (3). Until only recently, the histogenesis of GCTs were controversial, but due to electronic microscopy and immunohistochemistry (IHC), it was possible to consider a peripheral nerve-related cell origin for these tumors $(4,5)$.

GCTs are rare lesions which can occur anywhere in the body. Lack et al conducted a retrospective study over a 32-year period and found that GCTs accounted for only $0.03 \%$ of more than 410,000 surgical specimens examined in their facility. They also found that these tumors involved more frequently the oral cavity, skin and subcutaneous tissue (6).

One year later, Johnston and Helwig published a study of 75 GCTs found in the GI tract, most of them being located in the esophagus, followed by the colon, perianal region, stomach, small intestine and appendix (7).

Recently, Yasuda et al (2020) conducted a literature search in PubMed and Ichushi-Web for the term 'gastric granular cell tumor' and found 71 cases reported from inception to 2019 (8). This reconfirms the rare appearance of gastric GCTs and, to the best of our knowledge, this is the 72 nd case described in the literature.

According to Patti et al gastric GCTs are more commonly diagnosed between the fourth to sixth decade of life, with no gender predisposition, but with ethnic concerns, with most cases reported in the Japanese population (10). In our case, the patient was a 52-year-old woman from Eastern Europe, whose age corresponds to the most common age range described in the literature for this type of tumor.

Most GI-GCTs are discovered incidentally on endoscopy. Some authors consider that it is doubtful whether symptoms such as dysphagia or abdominal discomfort are caused by GCTs due to their small size, with the exception of large gastric GCTs, which may be directly responsible for specific symptoms such as gastric obstruction or upper GI hemorrhage (10). Regarding our case, the localization of the tumor in the gastric cardia could have caused the abdominal discomfort, without dysphagia or other distinct symptoms; a hypothesis confirmed by the remission of the symptoms after tumor excision.
Conventional endoscopy is the most frequent technique performed for the evaluation of the GI tract. For this reason, GI-GCT is usually discovered upon endoscopy and it is often described as a submucosal mass with overlying normal mucosa, on occasion with yellow discoloration (11). On the other hand, if available, endoscopic ultrasound (EUS) is the best procedure to evaluate GI-GCTs which are usually described as hypoechoic, homogeneous and smooth-edged lesions. Nonetheless, GI-GCTs can be hyperechoic, heterogeneous and have irregular margins as well. Most importantly, EUS can show whether the tumor involves or originates from muscularis propria (9).

Histologically, most gastric GCTs develop in the submucosal layer, being composed of sheets or compact nests of polygonal and fusiform cells with abundant, eosinophilic or amphophilic granular cytoplasm, with Periodic acid-Schiff (PAS)-positive granules and uniform pyknotic nuclei. The granular aspect of these tumors, positivity for CD68 and PAS, are due to the accumulation of lysosomes in the cell cytoplasm $(12,13)$. Lazar and Fletcher showed that CD68 is positive in nonneural GCTs as well (12).

We noted strong expression of S100 protein and CD56 which was concordant with results from previous studies and supports the neural origin of this tumor $(11,14)$.

Inhibin is a hormone secreted primarily by ovarian granulosa cells and testicular Sertoli cells, with involvement in the regulation of the pituitary-gonadal feedback system. Inhibin alpha is a useful marker for ovarian sex cord-stromal tumors and adrenocortical neoplasm. Some studies have reported expression of inhibin alpha in GCTs with different locations, including those with GI origin $(11,13,14)$. Vered et al studied the expression of inhibin alpha (ABD Serotec, Clone R1, dilution 1:50) in 42 oral GCTs and 38 of them were diffusely positive (13). An et al analyzed the inhibin alpha expression (ABD Serotec, MCA951S, dilution 1:100) in 58 cases of GI-GCTs, 30 of them being positive (52\%), with more common expression in colorectal than esophageal GCTs. Only 1 out of 3 gastric GCTs was positive (11). In contrast, Parfitt et al reported no expression of inhibin alpha (Cell Marque, dilution 1:25) in 7 GI-GCTs (15).

Fanburg-Smith et al six histologic criteria of malignancy in their 73 cases of GCT which included: necrosis, spindling of tumor cells, increased nuclear-to-cytoplasmic ratio, nuclear pleomorphism, vesicular nuclei with prominent nucleoli and an increased mitotic rate with more than two mitoses/10 high-power fields (x200 magnification). Based on these criteria, they classified GCTs into benign (none of the criteria or focal pleomorphism), atypical (1 or 2 criteria) and malignant (3 to 6 criteria) (16). Even if in our case there was a small population of spindle cells within the tumor, it was insufficient in our opinion to define it as atypical; thus, we reported the tumor as a benign GCT. To the best of our knowledge, only two cases of malignant gastric GCT have been reported in the English literature; both patients were diagnosed in Japan (8).

The Ki-67 proliferation index and p53 expression have been correlated with histologic classification and clinical course. Benign GCTs are commonly negative for p53 and the Ki-67 index is under $10 \%$, while malignant GCTs show upregulation of p53 as well as a Ki-67 index higher than 10\% (10). In our case presented above, the Ki-67 index was lower than $1 \%$, which supported the benign character of the tumor. 
Table I. Antibodies used to establish the IHC profile of the tumor.

\begin{tabular}{|c|c|c|c|c|}
\hline Antibody & IHC expression & Dilution & Clone & Company \\
\hline S100 & Positive & RTU & Polyclonal & Novocastra \\
\hline Inhibin alpha & Positive & RTU & R1 & Dako/Agilent Technologies, Inc. \\
\hline CD56 & Positive & $500: 1$ & CD564 & Novocastra \\
\hline CD68 & Positive & RTU & PG-M1 & Dako/Agilent Technologies, Inc. \\
\hline Ki-67 & $<1 \%$ & RTU & MIB 1 & Dako/Agilent Technologies, Inc. \\
\hline CD34 & Negative & RTU & QBEnd 10 & Novocastra \\
\hline CD117 & Negative & RTU & EP10 & Master Diagnostica \\
\hline DOG1 & Negative & RTU & K9 & Novocastra \\
\hline PanCK & Negative & RTU & AE1/AE3 & Dako/Agilent Technologies, Inc. \\
\hline Calretinin & Negative & RTU & DAK-Calret 1 & Dako/Agilent Technologies, Inc. \\
\hline
\end{tabular}

RTU, ready to use; IHC, immunohistochemistry.

A

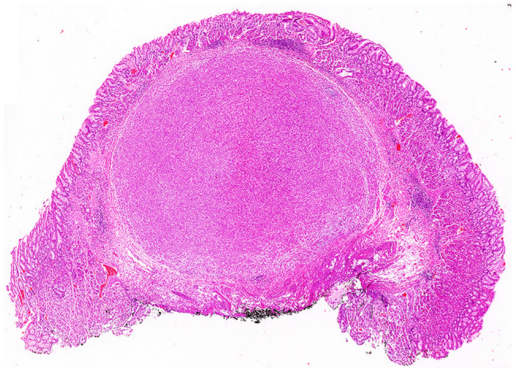

C

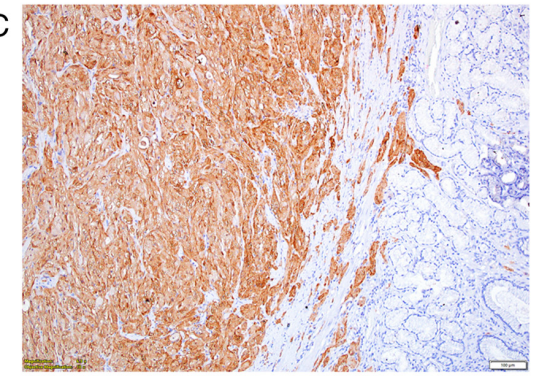

E

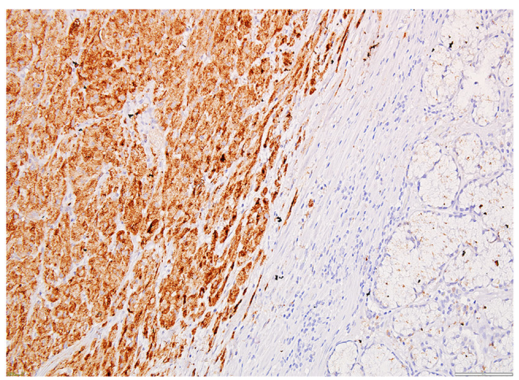

B

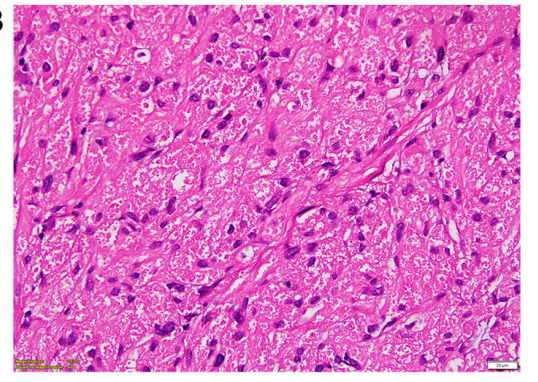

D

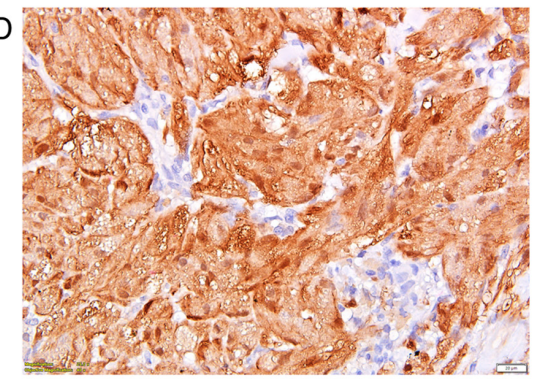

$\mathrm{F}$

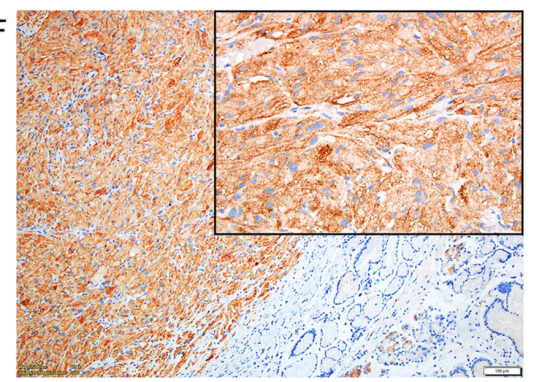

Figure 1. (A) Low power view of the granular cell tumor arising in the submucosa, covered by gastric mucosa (H\&E, original magnification x1). (B) High power view of the tumor cell's granular cytoplasm (H\&E, original magnification $\mathrm{x} 40$ ). Immunohistochemically, tumor cells showed strong expression of (C and D) S100 protein (C, original magnification x10; D, original magnification x40), (E) inhibin alpha (original magnification x20) and (F) CD56 (magnification x10; inset $\mathrm{x} 40)$.

The best course of treatment for GI-GCTs is resection by ESD. Lu et al published a study on 14 patients with esophageal GCTs treated by ESD. This research showed that this procedure removes the tumor entirely and also provides all the means necessary for pathological assessment. Moreover, patient follow-up ranged from 4 to 40 months, during which no local recurrence was observed (17).
In conclusion, a GCT arising in the stomach is a rare condition, mostly benign, which should be taken into consideration when a gastric polypoid lesion is examined. Inhibin alpha, along with S100 protein, prove to be valuable in the differential diagnosis of GCTs from other tumors, but further studies should be performed to determine why tumor cells show immunohistochemical expression for inhibin alpha. 


\section{Acknowledgements}

Not applicable.

\section{Funding}

Article processing charge for publication was supported by 'Victor Babeș' University of Medicine and Pharmacy (Timişoara, Romania).

\section{Availability of data and materials}

All information in this article is documented by relevant references, and further information concerning the case report is available upon request.

\section{Authors' contributions}

SMT contributed to the conception of the study, collected, analyzed and interpreted data from the literature and critically revised the manuscript. RAB contributed to the conception of the study, performed the literature research, drafted the manuscript and is responsible for confirming the authenticity of all the raw data. ADP contributed to the conception of the study, performed the literature research, drafted the manuscript and is responsible for confirming the authenticity of all the raw data ALCD contributed to the interpretation of the data from the literature, collected, analyzed and interpreted the data corresponding to the patient and critically revised the manuscript. IMR collected, analyzed and interpreted the data corresponding to the patient and critically revised the manuscript. OP performed the literature research, selected the included studies, analyzed and interpreted the data and drafted the manuscript. All authors read and approved the final manuscript.

\section{Ethics approval and consent to participate}

Ethics approval is not required for case reports at our institution. Informed consent from the patient was obtained to utilize images of the tissue for scientific purposes, with the condition of not disclosing identifiable information.

\section{Patient consent for publication}

The patient provided consent for publication.

\section{Competing interests}

The authors declare that they have no conflicts of interest or competing interests.

\section{Authors' information}

Sorina Maria Taban: ORCID: 0000-0002-3971-2756 Robert Alexandru Barna: ORCID: 0000-0003-4634-969X Alis Liliana Carmen Dema: ORCID: 0000-0003-0767-2718 Iulia Maria Ratiu: ORCID: 0000-0002-5622-9926 Oana Popa: ORCID: 0000-0003-3883-2438 Andrei Dorel Plopeanu: ORCID: 0000-0002-4900-4809.

\section{References}

1. Weber CO: Anatomical examination of a hypertrophic tongue along with remarks on the formation of striated muscle fibers. Virchows Arch A Pathol Anat 7: 115-125, 1854 (In German).

2. Abrikossoff A: Concerning myomas starting from the striated voluntary musculature. Virchows Arch Pathol Anat 260: 215-233, 1926 (In German).

3. Kim DJ, Kim HW, Park SB, Choi CW, Kang DH, Hong JB, Ji BH and Lee CS: A case of gastric granular cell tumor: Review of literature and features of endoscopic ultrasonography. Korean J Helicobacter Up Gastrointest Res 15: 59, 2015.

4. Fisher ER and Wechsler H: Granular cell myoblastoma-a misnomer. Electron microscopic and histochemical evidence concerning its schwann cell derivation and nature (granular cell schwannoma). Cancer 15: 936-954, 1962.

5. Mazur MT, Shultz JJ and Myers JL: Granular cell tumor. Immunohistochemical analysis of 21 benign tumors and one malignant tumor. Arch Pathol Lab Med 114: 692-696, 1990.

6. Lack EE, Worsham RGF, Callihan MD, Crawford BE, Klappenbach S, Rowden G and Chun B: Granular cell tumor: A clinicopathologic study of 110 patients. J Surg Oncol 13: 301-316, 1980.

7. Johnston MJ and Helwig EB: Granular cell tumors of the gastrointestinal tract and perianal region A study of 74 cases. Dig Dis Sci 26: 807-816, 1981.

8. Yasuda A, Yasuda T, Imamoto H, Hiraki Y, Momose K, Kato H, Iwama M, Shiraishi O, Shinkai M, Imano M, et al: A case of a gastric granular cell tumor preoperatively diagnosed and successfully treated by single-incision laparoscopic surgery. Surg Case Rep 6: 44, 2020.

9. Barakat M, Kar AA, Pourshahid S, Ainechi S, Lee HJ, Othman M and Tadros M: Gastrointestinal and biliary granular cell tumor: Diagnosis and management. Ann Gastroenterol 31: 439-447, 2018.

10. Patti R, Almasio PL and Di Vita G: Granular cell tumor of stomach: A case report and review of literature. World J Gastroenterol 12: 3442-3445, 2006.

11. An S, Jang J, Min K, Kim MS, Park H, Park YS, Kim J, Lee JH, Song HJ, Kim KJ, et al: Granular cell tumor of the gastrointestinal tract: Histologic and immunohistochemical analysis of 98 cases. Hum Pathol 46: 813-819, 2015.

12. Lazar AJF and Fletcher CDM: Primitive nonneural granular cell tumors of skin: Clinicopathologic analysis of 13 cases. Am J Surg Pathol 29: 927-934, 2005.

13. Vered M, Carpenter WM and Buchner A: Granular cell tumor of the oral cavity: Updated immunohistochemical profile. J Oral Pathol Med 38: 150-159, 2009.

14. Fine SW and Li M: Expression of calretinin and the alpha-subunit of inhibin in granular cell tumors. Am J Clin Pathol 119: 259-264, 2003.

15. Parfitt JR, McLean CA, Joseph MG, Streutker CJ, Al-Haddad S and Driman DK: Granular cell tumours of the gastrointestinal tract: Expression of nestin and clinicopathological evaluation of 11 patients. Histopathology 48: 424-430, 2006.

16. Fanburg-Smith JC, Meis-Kindblom JM, Fante $R$ and Kindblom LG: Malignant granular cell tumor of soft tissue: Diagnostic criteria and clinicopathologic correlation. Am J Surg Pathol 22: 779-794, 1998.

17. Lu W, Xu MD, Zhou PH, Zhang YQ, Chen WF, Zhong YS and Yao LQ: Endoscopic submucosal dissection of esophageal granular cell tumor. World J Surg Oncol 12: 221, 2014.

This work is licensed under a Creative Commons Attribution-NonCommercial-NoDerivatives 4.0 International (CC BY-NC-ND 4.0) License. 